Revista Perspectivas Online: Biológicas \& Saúde Junho/2021, v.11, n.37, p.62-70 ISSN: 2236-8868 (Online)

DOI: $10.25242 / 8868113720212291$

\title{
CONSUMO DE ALIMENTOS ULTRAPROCESSADOS EM PROFISSIONAIS DE SAÚDE DURANTE O COVID-19
}

\author{
Arthur Moraes Lamounier ${ }^{1 *}$, Bruna Thamara Gonçalves Frões ${ }^{1}$, Larissa de Macedo Rocha \\ Barbosa ${ }^{1}$, Letícia Ferrão de Oliveira ${ }^{1}$, Matheus Filipe Torres ${ }^{1}$, Mônica Thais Soares \\ Macedo $^{2}$ \& Josiane Santos Brant Rocha ${ }^{1}$
}

\section{RESUMO}

LAMOUNIER, A.M.; FRÕES, B.T.G.; BARBOSA, L.M.R.; OLIVEIRA, L.F.; TORRES, M.F.; MACEDO, M.T.S.; ROCHA, J.S.B. Consumo de alimentos ultraprocessados em profissionais de saúde durante o COVID-19. Perspectivas Online: Biológicas \& Saúde, v.11 , n.37, p.62-70, 2021.

O consumo exacerbado de alimentos ultraprocessados está associado a um aumento das taxas de obesidade, favorecendo a prevalência de doenças crônicas não transmissíveis. Nesse sentido, o objetivo do estudo foi investigar a prevalência do consumo de alimentos ultraprocessados em profissionais atuantes na Atenção Primária durante a pandemia do COVID-19 em Montes Claros, Minas Gerais. Trata-se de uma pesquisa descritiva, com profissionais de saúde atuantes na atenção primária de Montes Claros. A coleta de dados ocorreu por meio de um questionário abordando o consumo de alimentos ultraprocessados, além das variáveis sociodemográficas, hábitos comportamentais e perfil antropométrico, disponibilizado no google forms no período de setembro a outubro de 2020. Foi utilizado análise descritiva para estimara prevalência da variável desfecho e associação verificada por análise bivariada considerando $\mathrm{p}<0,05$. Foram entrevistados 210 profissionais de saúde, destes a maioria era do sexo feminino $(75,7 \%)$, tinham companheiros $(71,4 \%)$, era de cor não branca $(57,6 \%)$, possui residência na área da saúde $(50,5 \%)$, atuava há mais de 5 anos $(95,7 \%)$ e apresentava outro tipo de modalidade contratual $(61,4 \%)$. A prevalência do consumo de alimentos ultraprocessados nesse período foi considerada elevada $(41 \%)$. A prevalência do consumo de alimentados ultraprocessados aponta para a necessidade de ações de promoção de saúde aos profissionais.

Palavras-chave: Alimento Industrializado; Obesidade; Pandemia; Isolamento Social.

\footnotetext{
'Centro Universitário FipMoc, Av, Profa. Aida Mainartina Paraiso, 80, Ibituruna, Montes Claros, MG, CEP: 39408-007, Brasil. ${ }^{2}$ Universidade Estadual de Montes Claros, Av. Prof. Rui Braga, s/n, Vila Mauricéia, Montes Claros, MG, CEP: 39401 089, Brasil.

(*) e-mail: arthurm.lamounier13@gmail.com

Data de recebimento: 10/ 03/2021 . Aceito para publicação: 21/06/2021.

Data de publicação: 30/06/2021.
}

Persp. Online: biol. \& saúde, Campos dos Goytacazes, 37 (11) 62-70, 2021

seer.perspectivasonline.com.br 
Revista Perspectivas Online: Biológicas \& Saúde June/2021, v. 11 , n. 37 , p.62-70

ISSN: 2236-8868 (Online)

DOI: $10.25242 / 8868113720212291$

\title{
CONSUMPTION OF ULTRAPROCESSED FOOD IN HEALTHCARE PROFESSIONALS DURING COVID-19
}

\author{
Arthur Moraes Lamounier ${ }^{1 *}$, Bruna Thamara Gonçalves Frões ${ }^{1}$, Larissa de Macedo Rocha \\ Barbosa ${ }^{1}$, Letícia Ferrão de Oliveira1, Matheus Filipe Torres ${ }^{1}$, Mônica Thais Soares \\ Macedo $^{2}$ \& Josiane Santos Brant Rocha ${ }^{1}$
}

\begin{abstract}
LAMOUNIER, A.M.; FRÕES, B.T.G.; BARBOSA, L.M.R.; OLIVEIRA, L.F.; TORRES, M.F.; MACEDO, M.T.S.; ROCHA, J.S.B. Consumo de alimentos ultraprocessados em profissionais de saúde durante o COVID-19. Perspectivas Online: Biológicas \& Saúde, v.11 , n.37, p.62-70, 2021.
\end{abstract}

ABSTRACT
$\begin{aligned} & \text { The exacerbated consumption of ultra- } \\ & \text { processed foods is associated with an }\end{aligned}$
$\begin{aligned} & \text { 2020. Descriptive analysis was used to } \\ & \text { increase in obesity rates, favoring the } \\ & \text { prevalence of chronic non- }\end{aligned}$ variable and association verified by
communicable diseases. In this sense,
bivariate analysis considering p <0.05.
anthropometric profile, available on

Keywords: Foods Ultra-Processed; Obesity; Pandemic; Social Isolation.

\footnotetext{
${ }^{1}$ FipMoc University Center, Av, Profa. Aida Mainartina Paraiso, 80, Ibituruna, Montes Claros, MG, CEP: 39408-007, Brazil. 2 Montes Claros State University, Av. Rui Braga, s/n, Vila Mauricéia, Montes Claros, MG, Postal Code: 39401-089, Brazil. (*) e-mail: arthurm.lamounier13@gmail.com Received: 03/10/2021. 


\section{INTRODUÇÃO}

Desde 26 de fevereiro de 2020, o novo coronavírus Sars-CoV-2, causador da COVID19, instalou-se de maneira oficial no Brasil em decorrência de uma série de fatores tais como a falta de informação científica a respeito do assunto, acarretando em um aumento exponencial no número de casos. Desde então, diversas ações políticas foram implementadas, a fim de restringir a circulação do vírus na sociedade, sendo a principal delas o isolamento social imposto a milhões de brasileiros, caracterizada pela paralisação de todas as atividades econômicas não essenciais (lockdown), com o intuito de manter as pessoas o maior tempo possível dentro de suas casas (STEELE et al., 2020).

Hábitos individuais foram completamente modificados, resultantes desse distanciamento social, chamando atenção principalmente para os hábitos alimentares (STEELE et al., 2020). Em um estudo realizado nos Estados Unidos, observou-se que desde o início da pandemia ocorreu um aumento no consumo de alimentos ultra processados pelos americanos, como batata frita, chocolate, refrigerante e sanduíches, ou seja, alimentos com uma alta densidade energética (MALTA; GRACIE, 2020).

Entende-se que a fabricação de alimentos ultra processados esteja diretamente relacionado ao projeto de uma refeição pronta, durável, acessível e altamente lucrativa, já que o seu custo de produção é muito reduzido, em função de suas características nutricionais, oriundas do processamento sintético de óleos, gorduras, açúcares, amido e proteínas. Diante disso, é de se esperar que o consumo exacerbado dessa qualidade de alimento esteja associado a um aumento das taxas de obesidade, favorecendo a prevalência de doenças crônicas não transmissíveis (DCNT) (OLIVEIRA et al., 2019).

Além do fato de a obesidade ser, por si só, um fator de risco para o desenvolvimento de DCNT, como a hipertensão arterial, câncer, diabetes mellitus tipo 2 e doenças cardiovasculares, o Centro de Controle e Prevenção de Doenças (CDC) dos Estados Unidos identificou a obesidade como condição comum entre os pacientes hospitalizados, sendo que, de 180 adultos hospitalizados, 48,3\% apresentavam essa condição. Com isso, observa-se que o excesso de peso, bem como as suas implicações, está associado a um pior prognóstico e a um aumento da mortalidade em pacientes acometidos pela COVID-19 (MOREIRA; REIS; FREIRE; 2020).

Sendo assim, segundo Misumi et al. (2019), a manutenção de uma prática alimentar saudável, com reduzida ingestão de alimentos ultra processados pode auxiliar na prevenção e no tratamento da COVID-19, por meio de dois mecanismos: pelo suprimento de macro e micronutrientes fundamentais para o fortalecimento do sistema imunológico e pela manutenção de um adequado volume de tecido adiposo no indivíduo, já que tanto o excesso quanto a carência estão associados a uma pior evolução da doença (DUTRA et al., 2020).

Dentre as populações adultas, os trabalhadores constituem um grupo exposto a hábitos alimentares inadequados devido a diversas situações que impossibitam a escolha dos aliementos saudáveis para as refeições. Dessa forma, podendo prejudicar o processo saúdedoença e, consequentemente, acarretando em possíveis implicações como afastamentos, gastos com tratamentos e perca da produtividade no trabalho (CARVALHO et al., 2020).

Nesse sentido, a finalidade deste trabalho é estimar a prevalência do consumo de alimentos ultra processados em profissionais atuantes na atenção primária durante a pandemia do COVID-19 em Montes Claros, Minas Gerais.

Persp. Online: biol. \& saúde, Campos dos Goytacazes, 37 (11) 62-70, 2021

seer.perspectivasonline.com.br 


\section{METODOLOGIA}

O estudo foi conduzido como parte de um projeto denominado "Condições de saúde de profissionais da saúde da atenção primaria durante o período de isolamento social no enfrentamento à pandemia da COVID-19", desenvolvido por um Grupo de Pesquisa do Centro Universitário UNIFIPMOC, aprovado pelo Comitê de Ética em Pesquisa cujo parecer refere-se 4.075.365. A população alvo foi limitada aos cirurgiões dentistas, médicos e enfermeiros atuantes na atenção primária, de um grande centro do norte de Minas Gerais. Entende-se como Atenção Primária à Saúde (APS), a principal porta de entrada ao sistema, desse modo, sendo responsável porações que tangenciam a saúde de caráter individual ou coletivo. Essas ações promovem acesso a qualquer outra unidade funcional do sistema, à depender da necessidade de cada usuário. Vale ressaltar, portanto, a importância e a valorização da APS mediante sua responsabilidade pelo ordenamento do sistema e pela coordenação do cuidado (LAVRAS, 2011).

Antes do período da coleta de dados, conduziu-se um estudo piloto, com profissionais atuantes na atenção primaria, que não fizeram parte da amostra final. $O$ estudo piloto permitiu que fossem testados os questionários. Após a essa fase, foram realizados os ajustes no instrumento de coleta e a pesquisa foi iniciada.

A coleta de dados ocorreu por meio do Formulário Google Forms, on-line, onde se utilizou as redes sociais, de forma pública Whatsapp, como disseminadores do mesmo. O instrumento ficou disponível por 30 dias consecutivos, entre os dias 1 de setembro a 1 de outubro de 2020. Foi utilizado um questionário com questões obtidas de outros instrumentos validados referentes aos fatores sociodemográficos (sexo; estado civil; cor da pele; residência na área da saúde; cargo e modalidade contratual), hábitos comportamentais (prática de atividade física e comportamento sedentário) e perfil antropométrico (Indíce de Massa Corporal). No tópico que diz respeito a alimentação, compreende-se uma breve definição de alimentos ultraprocessados, que são alimentos cuja formulações industriais estão prontas para o consumo, a exemplo: bolachas salgadas e salgadinhos tipo chips; bolos, tortas, biscoitos doces, chocolates, balas; cereais matinais; hambúrgueres e salsichas; lanches do tipo fast food; pratos prontos ou semiprontos (pizzas, pratos de massa ou de carne congelados, macarrão instantâneo e sopas em pó); refrigerantes e outras bebidas açucaradas. A cerca disto, o atuante, precisaria pontuar o consumo desse tipo de alimento, sendo 0 em caso de aumento, 1 se permaneceu o mesmo, 2 para casos que houve uma diminuição e 3 quando não houve consumo.

Para análise do Índice de Massa Corporal (IMC) foi utilizado os valores de peso e estatura autorreferidos pelos entrevistados. A classificação do ponto de corte foi baseada nos pontos propostos pela World Health Organization (WHO) (1998), para a população adulta, adotando-se como referência os valores de $<18,5 \mathrm{~kg} / \mathrm{m}^{2}$ (baixo peso), entre 18,5 e $24,9 \mathrm{~kg} / \mathrm{m}^{2}$ (intervalo normal) e entre 25 e $29,9 \mathrm{~kg} / \mathrm{m}^{2}$ (sobrepeso). Posteriormente foi dicotomizado em normal $\left(<24,9 \mathrm{~kg} / \mathrm{m}^{2}\right)$ e alterado $\left(\geq 25 \mathrm{~kg} / \mathrm{m}^{2}\right)$.

Para análise dos dados, utilizou-se o programa estatístico SPSS (Statistical Package for the Social Sciences), versão 21, para realizar uma análise descritiva exploratória dos dados, com distribuição de frequências das variáveis do estudo.

\section{RESULTADOS}

Foram entrevistados 210 profissionais de saúde com média de idade de 36,7 $\pm 9,08$ anos, dos quais, $86(41 \%)$ teve um aumento do consumo de alimentos ultraprocesados, $95(45,2 \%)$ não

Persp. Online: biol. \& saúde, Campos dos Goytacazes, 37 (11) 62-70, 2021

seer.perspectivasonline.com.br 
teve alterações em relação ao consumo, $19(9,0 \%)$ registrou uma diminuição e por fim, $10(4,8 \%)$ não consumiram (Gráfico 1).

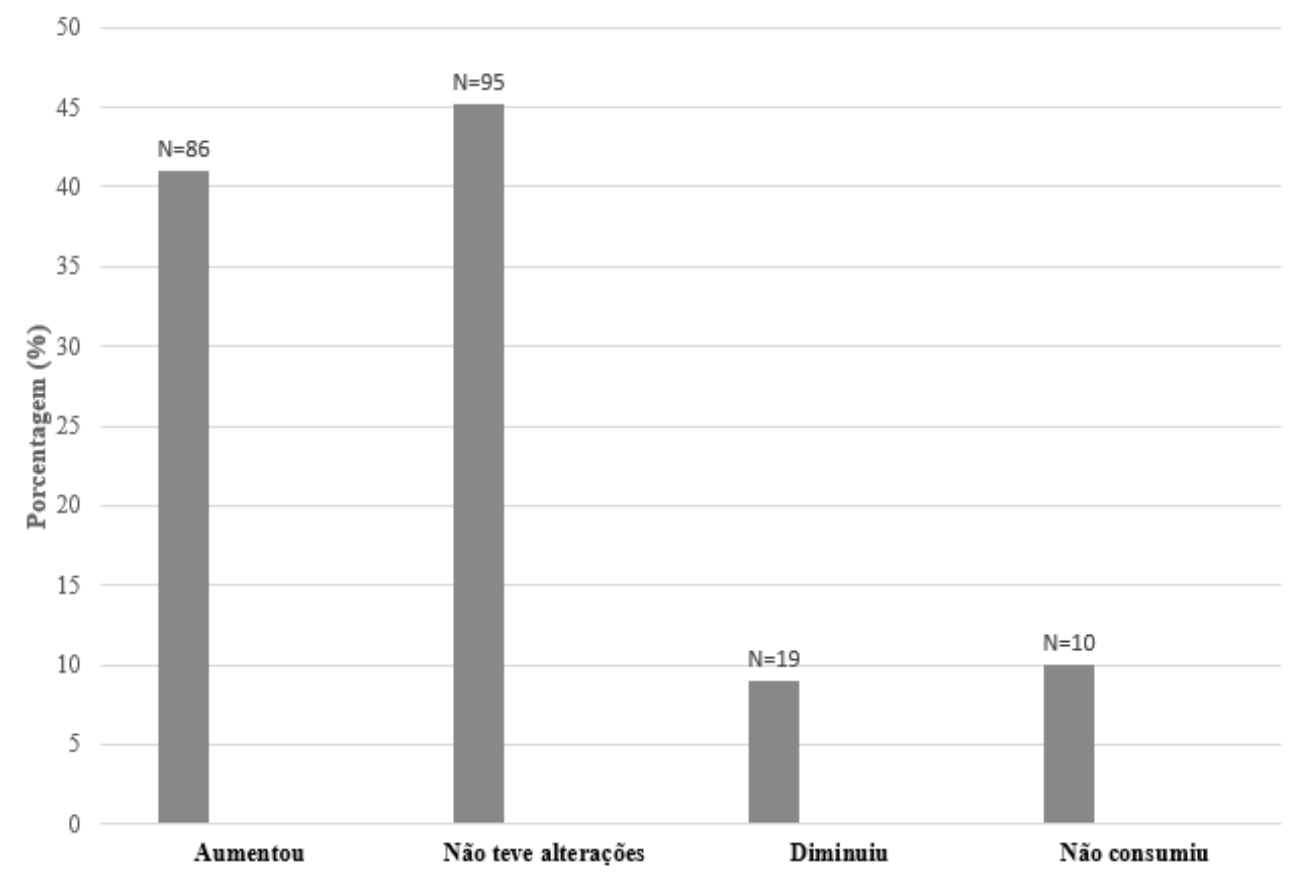

Gráfico 1: Consumo de alimentos ultraprocessados durante o isolamento social, devido a pandemia do COVID-19 dos profissionais da saúde atuantes na atenção primária de Montes

Claros-MG. 2020.

Quanto ao perfil da amostra investigada, mais da metade dos profissionais de saúde eram sexo feminino $(75,7 \%)$, tinha companheiros $(71,4 \%)$, era de cor não branca $(57,6 \%)$, possuía residência na área de saúde $(50,5 \%)$, atuava há mais de 5 anos $(95,7 \%)$ e apresentava outro tipo de modalidade contratual $(61,4 \%)$. Quanto aos hábitos comportamentais, a maioria dos entrevistados não realiza atividade física (59\%) e apresentou comportamento sedentário $(59,5 \%)$. Já sobre o perfil antropométrico, a maioria apresentava IMC normal $(53,3 \%)$ (Tabela 1$)$.

Tabela 1: Perfil sociodemográfico, hábitos comportamentais e perfil antropométrico dos profissionais da saúde atuantes na atenção primária de Montes Claros-MG. 2020.

\begin{tabular}{cccc}
\hline \multicolumn{2}{c}{ Variável } & n & \% \\
\hline Fatores sociodemográficos & & 51 & 24,3 \\
Sexo & Masculino & 159 & 75,7 \\
& Feminino & & \\
& Com & 150 & 71,4 \\
Estado Civil & companheiro & & 28,6 \\
& Sem & 60 & 42,4 \\
& companheiro & & 57,6 \\
Cor de pele & Branca & 121 & \\
& Não branca & & \\
& & &
\end{tabular}

Persp. Online: biol. \& saúde, Campos dos Goytacazes, 37 (11) 62-70, 2021

seer.perspectivasonline.com.br 


$\begin{array}{cccc}\begin{array}{c}\text { Residência na } \\ \text { área da saúde }\end{array} & \text { Possui } & 106 & 50,5 \\ \text { Nempo de } & \text { Não possui } & 104 & 49,5 \\ \begin{array}{c}\text { atuação } \\ \text { Até 4 anos }\end{array} & 9 & 4,3 \\ \text { Canoso } & \text { ou mais } & 201 & 95,7 \\ & \text { Médico } & 31 & 14,8 \\ & \text { Enfermeiro } & 52 & 24,8 \\ \text { Modalidade } & \text { Dentista } & 127 & 60,5 \\ \text { contratutal } & \text { Estatutária } & 81 & 38,6 \\ \text { Hábitos Comportamentais } & \text { Outros } & 129 & 61,4 \\ \text { Atividade física } & \text { Não } & 124 & 59,0 \\ & \text { Sim } & 86 & 41,0 \\ \text { Comportamento } & \text { Não } & 85 & 40,5 \\ \text { sedentário } & \text { Sim } & 125 & 59,5 \\ \text { Perfil antropométrico } & \text { Normal } & 112 & 53,3 \\ \text { IMC } & \text { Alterado } & 98 & 46,7 \\ & & & \end{array}$

\section{DISCUSSÃO}

Esse estudo teve como objetivo estimar a prevalência do consumo de alimentos ultraprocessados em profissionais atuantes na atenção primária durante a pandemia de COVID19. Os resultados evidenciaram que esses profissionais da saúde apresentaram modificações no consumo de alimentos ultraprocessados durante o isolamento social, visto que $41 \%$ dos entrevistados relataram ter aumentado o consumo e $9 \%$ diminui o consumo desses alimentos. Os alimentos do tipo ultraprocessados, são aqueles alimentos que necessitam de pouca ou de nenhuma preparação para se consumir e, com frequência, são compostos por grandesquantidades de açúcares, gorduras e, portanto, possuem um teor energético muito alto, quando comparado com os alimentos não processados ou minimamente processados (D'AVILA; KIRSTEN, 2017).

Ao observar as características da amostra estudada, entende-se que a maioria era do sexo feminino, não praticava atividade física, apresentando comportamento sedentário e Índice de Massa Corporal (IMC) elevado - conforme recomendações da World Health Organization (WHO, 1998). Dessa forma, padrões inadequados de hábitos alimentares e comportamentais podem ser relacionados a maior predisposição ao consumo de alimentos ultraprocessados. Nota-se que tais hábitos divergem das recomendações do Guia Alimentar para a População Brasileira, que, além de incentivarem hábitos de vida saudáveis, indicam o consumo de pequena quantidade de alimentos processados e contraindica o consumo de ultraprocessados (BRASIL, 2014).

Os Guias Alimentares para a População Brasileira (GAPB) tem como objetivo proporcionar a devida orientação alimentar, levando em consideração, para isso, aspectos biológicos, socioculturais e ambientais, de forma que doenças crônicas não transmissíveis sejam prevenidas e as comorbidades associadas reduzidas (OLIVEIRA; SANTOS, 2020).

Persp. Online: biol. \& saúde, Campos dos Goytacazes, 37 (11) 62-70, 2021

seer.perspectivasonline.com.br 
Nesse sentido, Girondoli et al. (2021) caracterizam o ambiente de trabalho como um lugar propício para a realização de atividades educativas em saúde, principalmente visando à promoção de uma alimentação saudável. Além disso, GAPB incentiva, ainda, o compartilhamento de habilidades culinárias - sendo essas definidas como as habilidades envolvidas em todas as etapas do preparo de alimentos - como forma de promoção de saúde.

Tendo em vista as recomendações do Guia Alimentar para a população Brasileira, bem como seus objetivos de promoção de saúde, nota-se que dietas com maior consumo de alimentos ultraprocessados possuem os piores perfis nutricionais, enquanto dietas baseadas em alimentos in natura ou minimamente processados são as mais indicadas (GABE; JAIME, 2020). O que se observa, entretanto, é que as mudanças no estilo de vida da população mundial e o avanço tecnológico da indústria alimentícia fortalecem o alto consumo de alimentos processados e ultraprocessados, contrariando as recomendações do GAPB (BESERRA et al.,2019).

Vale ressaltar, que o consumo persistente dessa qualidade de alimentos está diretamente vinculado ao principal fator de risco desencadeado pela alimentação exacerbada: a obesidade. Nesse sentido, essa condição é caracterizada como uma doença crônica não transmissível, sendo o problema nutricional de maior crescimento nas últimas décadas, tanto no Brasil, quanto no mundo (PIRES; GONÇALVES, 2021). Diante disso, a obesidade é considerada o principal fator de risco para uma grande variedade de outras doenças crônicas não transmissíveis, tais como o câncer, o diabetes mellitus e a hipertensão arterial, as quais apresentam grande período de latência, longo período de evolução, lesões irreversíveis e complicações graves, sendo que em estudos do Banco Mundial, as doenças crônicas não transmissíveis são responsáveis por uma taxa de 5 e 9 vezes maior de morte prematura do que as doenças transmissíveis, em homens e mulheres, respectivamente (MARIATH et al., 2007).

Isso acontece pelo fato de que a obesidade produz uma inflamação crônica no organismo, causando um desbalanço metabólico e imunológico e, com isso, gerar estresse oxidativo, disfunção da parede das artérias e anomalias cardiovasculares que poderiam levar o paciente a uma hiper reação inflamatória e, em casos de infecção por Sars-CoV-2, desencadear uma série de complicações associadas ao pior prognóstico da infecção por COVID-19 (SILVA, 2020).

Em suma, depreende-se que a Pandemia da COVID-19, enquanto fator isolado, influenciou no aumento do consumo de alimentos ultraprocessados por profissionais da saúde. Nesse sentido, o alto consumo desses alimentos é uma realidade, a nível mundial, independente de áreas profissionais ou faixa etária. Segundo Silva et al. (2020), há evidências de que a pandemia, apesar de não poder ser responsabilizada integralmente, agravou a situação de insegurança alimentar e nutricional no Brasil, que, conforme a Lei Orgânica de 2006, garante aos brasileiros o acesso regular e permanente a alimentos de qualidade, de modo que se instaurem práticas alimentares promotoras de saúde. Assim, evidencia-se que a COVID-19 dificulta a promoção de saúde pelas vias alimentares e nutricionais.

É oportuno considerar algumas limitações apresentadas após a realização dessa pesquisa. A primeira está relacionada ao fato de não ser possível verificar a veracidade das informações contempladas no questionário aplicado aos profissionais, uma vez que as respostas são subjetivas e não baseadas em parâmetros técnicos de avaliação nutricional. A segunda referese a não utilização de um questionário a respeito da quantidade de porções servidas desses alimentos, visto que esse fator pode ser considerado importante para análises futuras. No entanto, entende-se que as informações coletadas e analisadas proporcionadas por esse estudo

Persp. Online: biol. \& saúde, Campos dos Goytacazes, 37 (11) 62-70, 2021

seer.perspectivasonline.com.br 
sejam úteis, visto que a amostra investigada foi representativa da população estudada, chamando atenção, principalmente, para um fato de suma importância na qualidade de vida e de trabalho desses profissionais.

\section{CONCLUSÕES}

Conclui-se por meio desse estudo que a maioria dos profissionais da saúde apresentaram alteração no consumo de alimentos ultraprocessados durante a pandemia. No contexto de isolamento social em decorrência da COVID-19 nota-se um aumento desse tipo de alimento, o que é refletido diretamente de modo prejudicial na saúde da população que os consomem. Esse aumento pode estar vinculado a uma série de fatores. Dessa forma, ações afirmativas são necessárias de modo a conscientizar a população dos prejuízos causados pelo consumo inadequado dos alimentos ultraprocessados, com isso, podendo propiciar uma melhora adequada na qualidade de vida desses indivíduos.

\section{REFERÊNCIAS}

BESERRA, J.B.; SOARES, N.I.S.; MARREIROS, C.S.; CARVALHO, C.M.R.G.; MARTINS, M.C.C.; FREITAS, B.J.S.A.; SANTOS, M.M.; FROTA, K.M.G. Crianças e adolescentes que consomem alimentos ultraprocessados possuem pior perfil lipídico? Uma revisão sistemática. Ciência \& Saúde Coletiva, v.25, n.12, p. 4979 - 4989, 2020.

BRASIL. Ministério da Saúde. Secretaria de Atenção Básica. Departamento de Atenção Básica. Guia Alimentar para a população brasileira. 2 ed. Brasília: Ministério da Saúde; 2014. 156p.

CARVAlHO, V.N.C.; COUTO, A.N.; VITIELlO, I.P.; SEVERGNINI, C.; POHL, H.H. Consumo de alimentos processados/ ultraprocessados e in natura por adultos e sua relação com o estado nutricional. Revista Brasileira de Obesidade, Nutrição e Emagrecimento, v. 14, n. 84, p. 66-72, 2020.

D'AVILA, H.F.; KIRSTEN, V.R. Consumo energético proveniente de alimentos ultraprocessados por adolescentes. Revista Paulista de Pediatria. São Paulo, v.35, n.1, p.54-60, 2017.

DUTRA, A.F.O.; DIAS, A.D.C.; ARAÚJO, D.G.S.; SILVA, E.M.; SILVA, I.M.F.; GOMES, L.M.F. A importância da alimentação saudável e estado nutricional adequado frente a pandemia de Covid-19. Brazil Journal of Development., Curitiba, v.6, n.9, p.66464-66473, 2020.

GABE, K.T.; JAIME, P.C. Práticas alimentares segundo o Guia alimentar para a população brasileira: fatores associados entre brasileiros adultos, 2018. Epidemiologia e Serviços de Saúde, v. 29, n.1, 2020.

GIRONDOLI, Y.M.; ROSSETO, C.I.C.; SOARES, M.C.R.; PEREIRA, S.G. Incentivo às habilidades culinárias e ao consumo de comida de verdade como estratégia de educação alimentar e nutricional no ambiente de trabalho. Brazilian Journal of Health Review, v.4, n.2, p.7424-7432, 2021.

LAVRAS, C. Atenção Primária à Saúde e a Organização de Redes Regionais de Atenção à Saúde no Brasil. Revista Saúde e Sociedade. São Paulo, v. 20, n. 4, p. 867-874, 2011.

Persp. Online: biol. \& saúde, Campos dos Goytacazes, 37 (11) 62-70, 2021

seer.perspectivasonline.com.br 
MALTA, D.C.; GRACIE, R. A pandemia da COVID-19 e as mudanças no estilo de vida dos brasileiros adultos: um estudo transversal, 2020. Epidemiologia a Serviço da Saude, Brasília, v. 29 p. 2020407, 2020.

MARIATH, A.B.; SILVA, R.O.; SCHMITZ, P.; CAMPOS, I.C.; MEDINA, J.R.P.; KRUGER, R.M. Obesidade e fatores de risco para o desenvolvimento de doenças crônicas não transmissíveis entre usuários de unidade de alimentação e nutrição. Caderno Saúde Pública. Rio de Janeiro, v.23, n.4, p. 897-905, 2007.

MISUMI, I.; STARMER, J.; UCHIMURA, T.; BECK, M. A.; MAGNUSON, T.; WHITMIRE, J. K. Obesity expands a distinct population of $\mathrm{T}$ cells in adipose tissue and increases vulnerability to infection. Cell Reports. v. 27, n. 2, p. 514-24, 2019.

MOREIRA; G.S; REIS; L.B.S.M.; FREIRE, P.B. Obesidade e agravamento da COVID 19. Health residencies journal. v.1, n.6, 2020.

OLIVEIRA, I.K.F.; MACHADO, E.B.; SOUSA, R.R.; PAIVA, A.A. Consumo de alimentos ultraprocessados e obesidade abdominal em adolescentes universitários. Revista Eletrônica Acervo Saúde. Local, v.11, n.16, p. 2-8, 2019.

OLIVEIRA, M.S.S.; SANTOS, L.A. Guias alimentares para a população brasileira: Uma análise a partir das dimensões culturais e sociais da alimentação. Ciência \& Saúde Coletiva v. 25, n.7, 2020.

PIRES, I.G.; GONÇALVES, D.R. Consumo alimentar e ganho de peso de gestantes assistidas em unidades básicas de saúde. Brazilian Journal of Health Review, v. 4, n. 1, p. 128-146, 2021.

SILVA, J.N. Obesidade e Covid-19: Quais as Evidências?. Revista Artigos. Com, v. 21, p. 5346, 82020.

SILVA, R.C.R.; PEREIRA, M.; CAMPELLO, T.; ARAGÃO, E.; FERREIRA, A.J.F.; BARRETO, M.L.; SANTOS, S.M.C. Implicações da pandemia COVID-19 para a segurança alimentar e nutricional no Brasil. Ciência \& Saúde Coletiva, v.25, n7., p. 2504-2504, 2020.

STEELE, E.M.; RAUBER, F.; COSTA; C.S.; LEITE, M.A.; GABE, K.T; LOUZADA, M.L.C.; BERTAZZI, R.L.; MONTEIRO, C.A. Mudanças alimentares na coorte NutriNet Brasil durante a pandemia de covid-19. Revista Saúde Pública. v. 54, p. 91,2020.

WORLD HEALTH ORGANIZATION (WHO). Obesity: preventing and managing the global epidemic. Geneva: Progam of Nutrition, Family and Reproductive Health, 1998.

Persp. Online: biol. \& saúde, Campos dos Goytacazes, 37 (11) 62-70, 2021

seer.perspectivasonline.com.br 